\title{
Michael Knoche
}

\section{Was macht die Zusammenarbeit von Bibliotheken so schwierig?}

Stellt man sich die Person leibhaftig vor Augen, die mit dieser Festschrift geehrt wird, erscheint es völlig unverständlich, warum die Zusammenarbeit von Bibliotheken ein Problem sein soll. Gibt es einen Bibliothekar, der sich für die Zusammenarbeit von Bibliotheken untereinander und mit anderen Wissenschaftsund Kultureinrichtungen aufgeschlossener und begeisterungsfähiger gezeigt hätte als Thomas Bürger? Gibt es jemanden, der für seine Kollegen mehr Interesse aufgebracht und sie mehr ermutigt hätte? Eine Person, die anscheinend völlig frei von hässlichen Gefühlen wie Missgunst, Neid und Angst um den eigenen Status agiert? Die das Große und Ganze zum Leitmotiv ihres Handelns gewählt hat? Zu Recht haben die Herausgeber für seine Festschrift das Thema „Kooperation“ gewählt. Eine bibliothekarische Festschrift mit diesem Fokus hat es noch nicht gegeben, aber es hat auch noch keinen Thomas Bürger gegeben.

Und doch ... Selbst eine Persönlichkeit mit herkulischer Integrationskraft stößt im deutschen wissenschaftlichen Bibliothekswesen an Grenzen. Denn es hängt nicht alles von Menschen ab. Es gibt auch kooperationsfeindliche Strukturen und Entwicklungen, die mit gutem Willen der Betroffenen allein nicht zu verändern sind.

Dabei sind Bibliotheken wie wenige andere Institutionen auf Arbeitsteilung und Kooperation angelegt. ${ }^{1}$ Bibliotheken müssen sich abstimmen, welche Schwerpunkte sie in ihrer Sammlung von analogen und digitalen Medien setzen wollen: in Stadt, Region, Verbund, gegebenenfalls auch national und international. Sie müssen sich fragen, welche Dienstleistungen bei der Produktion von Metadaten gemeinsam erbracht und wie sie ihre Bestände in den globalen Nachweissystemen und Suchmaschinen sichtbar machen können. Sie müssen untereinander vereinbaren, wer die Langzeitverfügbarkeit der analogen und digitalen Medien garantiert. Alle Bibliotheksleistungen in Bestandsaufbau, Nachweis, physischer Aufbewahrung, Speicherung digitaler Daten und Informationsvermittlung können sinnvoll nur noch in abgestimmter Kooperation organisiert werden. Ihre Nutzer sind schon lange nicht mehr mit dem lokal verfügbaren Bestand zufriedenzustellen, sondern brauchen einen umfassenden Zugriff auf $\mathrm{Pu}$ -

1 Den folgenden Ausführungen liegen Gedanken zugrunde, die ich in dem Buch Die Idee der Bibliothek und ihre Zukunft (Göttingen: Wallstein 2018) für eine allgemein interessierte Leserschaft dargelegt habe.

๖ Open Access. (C) 2018 Michael Knoche, publiziert von De Gruyter. (c) BY-Nc-ND Dieses Werk ist lizenziert unter der Creative Commons Attribution-NonCommercial-NoDerivatives 4.0 Lizenz. 
blikationen. Waren es früher Sonderleistungen wie etwa die Fernleihe, die im Verbund der Bibliotheken untereinander erbracht wurden, sind es heute die Kernaufgaben, die in geteilter Verantwortung erledigt werden müssen. Die technologischen, finanziellen und organisatorischen Herausforderungen sind so groß, dass auch die mächtigsten Institutionen auf Zusammenarbeit angewiesen sind. Selbst die Library of Congress in Washington D. C. ist nicht mehr autonom zu denken.

So notwendig die Bibliotheken zusammenwirken müssen, so kompliziert sind die Voraussetzungen für kooperatives Handeln in Deutschland. Vor 25 Jahren wurden die Hochschulen einschließlich der Hochschulbibliotheken durch die Ministerialbürokratie noch aktiv gesteuert. Damals haben sich Minister in die Berufung von Professoren eingemischt. Heute ersetzen Zielvereinbarungen den Ministerwillen. So werden den Hochschulen nur die Rahmenbedingungen ihres Wirkens vorgegeben. Sie müssen sich im internationalen Wettbewerb des Wissenschaftsmarktes behaupten und um Studenten, qualifiziertes Lehrpersonal, Finanzmittel und Reputation konkurrieren. Jede Hochschule gibt sich ihr Leitbild und profiliert sich: als Ausbildungseinrichtung, als Anstalt zur Politikberatung oder als Exzellenzuniversität. Deutlicher als früher bekommen auch die Bibliotheken von ihren Hochschulen klare Vorgaben, was sie zum Erfolg der Gesamtinstitution beizutragen haben.

Dabei können die überregionalen Absprachen und Dienstleistungen der Bibliotheken, von denen die einzelne Hochschule nur mittelbar profitiert, ins Hintertreffen geraten. Es ist schon haushaltsrechtlich schwierig, Ausgaben zu tätigen, die nicht unmittelbar der eigenen Organisation zugutekommen oder zunächst nicht fühlbar werden. Die Hochschulleitungen sehen sich in ihrem Rollenverständnis den eigenen Hochschulangehörigen stärker verpflichtet als der Allgemeinheit und steuern die Bibliotheken entsprechend. ${ }^{2}$

Die Hochschulbibliotheken haben in ihrer abhängigen Stellung keine ausreichenden Handlungsspielräume. Wer aber kann dann Bibliothekspolitik gestalten? Fast alle möglichen Akteure fallen aus: Welches Bundesland verfolgt eine erkennbare Bibliothekspolitik? Alles steht unter dem Vorbehalt der Hochschulautonomie. Die Kultusministerkonferenz? Hier lässt sich nur schwer Einstimmigkeit herstellen, und falls doch, ist die Finanzministerkonferenz die nächste hohe Hürde. Stets muss Rücksicht auf das schwächste oder am wenigsten einsichtige Mitglied unter den Bundesländern genommen werden. Neoseparatistische Tendenzen sind nicht gänzlich zu übersehen. Die Hochschulrektorenkonferenz als Organ ist zu schwach und nicht für die Staats-, Regional-,

2 Vgl. Nettelbeck, Joachim: Das Wunschbild des starken Präsidenten. In: Frankfurter Allgemeine Zeitung vom 17. Februar 2016, S. N4. 
Forschungs- und Spezialbibliotheken zuständig. Es gibt in Deutschland keinen Akteur für nationale Bibliothekspolitik. Der Rat für Informationsinfrastrukturen, dessen Mitglied Thomas Bürger ist, macht sich seit 2014 nun darüber wenigstens kluge Gedanken. Die ersten Verlautbarungen zur Aufgabenverteilung beim Teilaspekt Forschungsdaten sind vielversprechend. ${ }^{3}$

Das Fehlen staatlichen Handelns führt unter anderem dazu, dass die deutschen Bibliotheken international einen schweren Stand haben. Die Musik spielt da, wo die Entscheidungswege zentraler und effizienter angelegt sind und Bibliotheken auf dem sich rasant verändernden Informationsmarkt wenigstens ansatzweise mithalten können: vor allem in den USA. Kein Wunder, dass sich die deutschen Bibliotheken dem Hostingdienst Portico anschließen wollen; zu umfassenden eigenen Lösungen fehlen ihnen die Möglichkeiten.

Die wichtigsten Akteure der nationalen Bibliothekspolitik sind in Deutschland kurioserweise die Wissenschaftsorganisationen, allen voran die Deutsche Forschungsgemeinschaft mit ihren Projektförderungen und der Wissenschaftsrat mit seinen Denkschriften und Evaluationen. Sie geben durchaus wichtige Impulse zur Weiterentwicklung der Informationsinfrastrukturen, aber sie können die Defizite nicht kontinuierlich und systematisch bearbeiten. Albert Bilo hat völlig recht, wenn er feststellt: „Zusätzlich benötigen die Anforderungen an eine wissenschaftliche Literaturversorgung nationale Rahmenbedingungen. Es ist nicht hinreichend, wenn dies auf die Ebene von Empfehlungen, Vorschlägen oder temporären Programmen beschränkt bleibt. “4

Da die Deutsche Forschungsgemeinschaft ein so wichtiger Bibliotheksakteur ist, ist es doppelt bedauerlich, dass gerade sie die Förderung eines Gemeinschaftsunternehmens eingestellt hat, das für das deutsche Bibliothekswesen jahrzehntelang wichtig und prägend war, das Sondersammelgebietsprogramm. Im Rahmen dieses überregionalen Systems waren 44 Staats- und Universitätsbibliotheken und zentrale Fachbibliotheken damit beauftragt, jeweils für einen der 120 Sammelschwerpunkte die wissenschaftliche Fachliteratur möglichst vollständig zu erwerben. Alle an diesem System beteiligten Bibliotheken hatten sich verpflichtet, ihre Spezialbestände deutschlandweit zur Verfügung zu stellen. Auf diese Weise sind einzigartig breite und tiefe Sammlungen von ana-

3 Vgl. Meinhardt, Haike: Informationsinfrastrukturen im Wandel. Empfehlungen und Diskussion des Rats für Informationsinfrastrukturen zum Forschungsdatenmanagement in Deutschland. In: Zeitschrift für Bibliothekswesen und Bibliographie 64 (2017) H. 5. S. 261-267.

4 Bilo, Albert: Was ist eigentlich Bibliothekspolitik? In: Buch, Bibliothek, Region. Wolfgang Schmitz zum 65. Geburtstag. Hrsg. von Christine Haug u. Rolf Thiele. Wiesbaden: Harrassowitz 2014. S. 191-205, hier S. 205. Vgl. auch den Beitrag von Pilzer, Harald: „Library politics“ und „Library policy“ im föderativen Staat. Eine Skizze. In: Buch, Bibliothek, Region. S. 321-340. 
logen und digitalen Medien in fast allen Wissenschaftsfächern entstanden, eine verteilte universale Sammlung.

Das Programm wurde nicht aus finanziellen Gründen aufgegeben. Die Einnahmen der Deutschen Forschungsgemeinschaft sind in den letzten Jahren kontinuierlich gestiegen. Aber der Anteil der Bibliotheksförderung ist zwischen 2005 und 2014 von 3,8 auf 1,9 \% gefallen. ${ }^{5}$ Vielmehr wurde der Sondersammelgebietsplan 2013 nach einer Evaluation mit schlechtem Ergebnis gestoppt. Das eigentliche Ziel des Programms wurde infrage gestellt, nämlich die Bildung eines möglichst vollständigen Literaturreservoirs. Daraufhin haben die Gremien der Forschungsgemeinschaft beschlossen, neue Informationsdienstleistungen „unter spezieller Berücksichtigung der Forschungsinteressen der jeweiligen Fachcommunities“ zu entwickeln. ${ }^{6}$ Die „Fachinformationsdienste“ sollen maßgeschneiderte Angebote für einzelne Disziplinen bereitstellen. Der Fokus der Förderung wurde also von der Sammlung auf die Informationsdienstleistung der Bibliotheken verlagert. Damit ist das fein geknüpfte Netz der überregionalen Bereitstellung von Publikationen in Deutschland gerissen. ${ }^{7}$ Nicht jedes Sondersammelgebiet wurde in einen Fachinformationsdienst überführt. Derzeit gibt es 38 Förderungen mit einem durchschnittlichen Volumen von jährlich einer Million Euro.

Was wird aus den bestehenden Spezialbeständen? Keine Einrichtung ist in der Lage, die vielen Zeitschriftenabonnements und die Sammlungen von Spezialliteratur aus eigener Kraft weiterzuführen. Soll jede einzelne Bibliothek zurückkehren zum Ideal einer Versorgung der gesamten „Universitas Litterarum“?

5 Vgl. Göttker, Susanne: Literaturversorgung in Deutschland. Von den Sondersammelgebieten zu den Fachinformationsdiensten. Wiesbaden: Dinges \& Frick 2016, S. 120. Die Verfasserin bietet eine gute Analyse der Veränderungen, die sich aus der neuen Förderpolitik ergeben.

6 Fachinformationsdienste: DFG stärkt Dienstleistungen der Bibliotheken für die Wissenschaft, Pressemitteilung der DFG vom 20. Dezember 2013. http://www.dfg.de/service/presse/ pressemitteilungen/2013/pressemitteilung_nr_54/index.html. Die Hintergründe der Entscheidung werden dargestellt von Lipp, Anne u. a.: „Die digitale Transformation weiter gestalten“ - Das Positionspapier der Deutschen Forschungsgemeinschaft zu einer innovativen Informationsinfrastruktur. In: Zeitschrift für Bibliothekswesen und Bibliographie 59 (2012) H. 6. S. 291-300. http://zs.thulb.uni-jena.de/servlets/MCRFileNodeServlet/jportal_derivate_ 00229529/j12-h6-auf-1.pdf. Alle aufgeführten Internetressourcen wurden zuletzt am 31. Oktober 2017 aufgerufen.

7 Mittler, Elmar: Nachhaltige Infrastruktur für die Literatur- und Informationsversorgung: im digitalen Zeitalter ein überholtes Paradigma - oder so wichtig wie noch nie? In: Bibliothek Forschung und Praxis 38 (2014) H. 3. S. 344-364. - Zusammenfassung der ganzen Debatte mit Stand vom Frühjahr 2015 bei Illig, Steffen: Von den SSG zu den FID. Eine kritische Reflexion von Anspruch und Wirklichkeit. In: Perspektive Bibliothek 4 (2015) H. 1. S. 5-28. http://dx.doi. org/10.11588/pb.2015.1.21644. 
Angesichts der vielen heterogenen Medien aller Fachgebiete und der immensen Kosten wäre eine solche Absicht realitätsfern. Oder soll sie versuchen, ihren traditionellen Schwerpunkt mit reduzierten Mitteln aufrechtzuerhalten? Sammlung, Speicherung und Vermittlung würden unweigerlich Torso bleiben. Soll sie auf jede eigene Sammlung verzichten und sich nur noch auf die Vermittlung konzentrieren? Das predigen die Digitalfundamentalisten schon lange: access versus ownership. Fragt sich nur, wer eigentlich liefern soll, wenn alle nach dem Prinzip handeln. Wer ist dann noch „owner“? ${ }^{8}$

In Anbetracht der erheblichen Konsequenzen dieses förderpolitischen Richtungswechsels wären viele Kollegen schon froh, wenn die Deutsche Forschungsgemeinschaft die Unterstützung der Fachinformationsdienste wenigstens mittelfristig garantierte. ${ }^{9}$ Denn für so weitreichende Aufgaben Mittel für nur drei Jahre $\mathrm{zu}$ bewilligen und 2018 schon wieder die ganze Programmlinie $\mathrm{zu}$ evaluieren, führt nur zu hektischer Betriebsamkeit der Beteiligten. (Hans Blumenberg hätte diesen Übereifer eine „,auf Überstürzung angelegte Erfüllungsversessenheit“" genannt. ${ }^{10}$ ) Die zeitliche Begrenzung bedeutet, ganz abgesehen von den Investitionen in den Aufbau der Dienste, dass auch Arbeitsverträge der hoch spezialisierten Mitarbeiter, die schwer zu bekommen und schwer zu halten sind, nur auf kurze Zeit befristet abgeschlossen werden können. Die Projektstruktur ist für diese Aufgaben völlig ungeeignet.

Vielleicht ist die Deutsche Forschungsgemeinschaft in der Frage der verteilten Sammelschwerpunkte auch der falsche Ansprechpartner. Gemäß ihrer Satzung kann sie mithelfen, übergreifende Informationsinfrastrukturen zu etablieren, aber sie kann Dienstleistungen nicht auf Dauer finanzieren. Man kann ihr nur den Vorwurf machen, ein bewährtes System fallengelassen zu haben, ohne dass eine Auffanglösung in Sicht war. Das Bundesforschungsministerium hüllt sich in dröhnendes Schweigen.

Das Programm „Sammlung Deutscher Drucke“ war ein anderer Anlauf, eine gemeinsame Erwerbungsstrategie der deutschen Bibliotheken zu etablieren. Im Jahr 1989, leider knapp vor der Wiedervereinigung, hat die Volkswagen-Stiftung auf Anregung Bernhard Fabians fünf großen westdeutschen Bibliotheken

8 Vgl. Knoche, Michael (Hrsg.): Die Zukunft des Sammelns an wissenschaftlichen Bibliotheken. Wiesbaden: Harrassowitz 2017; Knoche, Michael: Haben Bibliotheken noch einen Sammelauftrag? In: Knoche, Michael: Auf dem Weg zur Forschungsbibliothek. Studien aus der Herzogin Anna Amalia Bibliothek. Frankfurt am Main: Klostermann 2016. S. 47-53. https://www. o-bib.de/article/view/2015H4S78-84/3326.

9 Vgl. Die Zukunft des Sammelns an wissenschaftlichen Bibliotheken. Eine Diskussion in der Herzogin Anna Amalia Bibliothek Weimar am 27.4.2016. In: Zukunft des Sammelns (wie Anm. 8). S. $125-137$.

10 Blumenberg, Hans: Lebenszeit und Weltzeit. Frankfurt am Main: Suhrkamp 1986, S. 175. 
(Bayerische Staatsbibliothek München, Herzog August Bibliothek Wolfenbüttel, Niedersächsische Staats- und Universitätsbibliothek Göttingen, Universitätsbibliothek Johann Christian Senckenberg Frankfurt am Main, Staatsbibliothek zu Berlin) fünf Jahre lang jeweils eine Million DM zur Verfügung gestellt. Ziel war es, alle aus dem deutschen Sprachraum und die im Ausland in deutscher Sprache erschienenen Druckwerke retrospektiv zu sammeln, $d$. h. fehlende Bücher antiquarisch in Originalausgaben zu erwerben, denn die historischen Bestände auch der besten Bibliotheken sind noch erstaunlich lückenhaft. Mit dem Programm sollte ein Ausgleich für das Fehlen einer historisch gewachsenen Nationalbibliothek geschaffen werden. Die Initiative war segensreich, aber sie hat an Schwung verloren. Heute wenden die fünf Bibliotheken und die Deutsche Nationalbibliothek zusammen nur noch etwa 800000 Euro für antiquarische Käufe in diesem Rahmen auf.

Kann eine umfassende Bereitstellung von Publikationen in unserem föderal verfassten Land wirklich anders organisiert werden als über verteilte Ressourcen? Erstrebenswert wäre ein modernisiertes „Sondersammelgebietsprogramm“. Schlimmstenfalls, wenn die Bibliotheken mit dieser Herausforderung alleingelassen werden und keine Hilfe bekommen, müssten sie selber die Aufgaben untereinander verteilen - mit dem Nachteil geringerer Verbindlichkeit und geringeren Leistungsvermögens. Außerdem würden dann die bestehenden Fachinformationsdienste, jeweils für drei Jahre von der Deutschen Forschungsgemeinschaft bewilligt, quer zu den langfristigen Absprachen der Bibliotheken liegen.

Es gab in Deutschland für die Bibliotheken früher zwei zentrale Dienstleistungsbereiche mit nennenswerter Ausstattung. Einen davon gibt es noch: die Ebene der Bibliotheksverbünde. Die sechs Verbundzentralen mit insgesamt etwa 200 Personalstellen wurden von den Bundesländern vor allem im Hinblick auf die gemeinsame Katalogisierung und Fernleihe ihrer Region gegründet. So betreibt etwa das kleine Land Hessen für seine 25 wissenschaftlichen Bibliotheken das Hessische Bibliotheksinformationssystem. Bei der Einrichtung dieser Strukturen, im letzten Viertel des 20. Jahrhunderts, entschied man sich leider gegen eine zentrale Datenhaltung im deutschen Bibliothekswesen.

Die Basisdienste für die digitale Bibliothek leisten die Verbünde fast alle in gleicher Weise. Die Deutsche Forschungsgemeinschaft und der Wissenschaftsrat halten daher die Zeit für eine grundlegende Reform gekommen, kritisieren das Regionalprinzip und empfehlen eine klare Aufgabenteilung. ${ }^{11}$ Der gemeinsamen Empfehlung von 2011 sind jedoch wenige Taten gefolgt. Die regional definierten Strukturen sind noch lange nicht überwunden und nicht nach Auf-

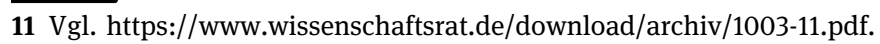


gaben sortiert. Hier steckt ein Potenzial für innovative Dienste, die für die überregionale Informationsinfrastruktur so dringend benötigt werden.

Die andere Dienstleistungsinstanz war bis 1999 das Deutsche Bibliotheksinstitut in Berlin. Die Anstalt des öffentlichen Rechts, zu 70 \% von den Ländern und $30 \%$ vom Bund finanziert, hatte Mitte der 1990er Jahre etwa 140 Stellen und einen Etat von 20 Millionen DM. Sie wurde durch den Wissenschaftsrat evaluiert, obwohl es keine wissenschaftliche, sondern eine Serviceeinrichtung war. Das Resultat war schlecht. Statt jedoch ihre Sachaufgaben und Dienstleistungen im Bereich Koordinierung, Beratung, Normierung und Innovation zu verbessern, wurde das Bibliotheksinstitut gleich ganz abgewickelt. Auch die Bibliothekare selber hatten ihren Anteil am Untergang. So wie es in der Politik heute wohlfeil ist, auf die Bürokraten in Brüssel zu schimpfen, haben Bibliotheksdirektoren gerne das Berliner Bibliotheksinstitut heruntergeputzt. Plötzlich aber war gar keine Koordinierungsinstanz mehr da. Es war dann auch keine Überraschung, dass es nicht gelang, eine Nachfolgeeinrichtung zu gründen, um die wichtigsten Dienste weiterzuführen. Die Wahrnehmung überregionaler Aufgaben des Bibliothekswesens hängt seither in der Luft.

Damit es aber nach außen nicht so dramatisch aussieht, wurde 2004 das dezentral organisierte Kompetenznetzwerk für Bibliotheken gegründet. Es wird von den Bundesländern gemeinschaftlich finanziert und umfasst viereinhalb Stellen. $\mathrm{Zu}$ den übrig gebliebenen Arbeitsbereichen gehören die Deutsche Bibliotheksstatistik, die EU- und Drittmittelberatung oder der Betrieb des Bibliotheksportals. ${ }^{12}$ Der Vorsitzende des Deutschen Bibliotheksverbandes klagt zu Recht: „Die Defizite sind erschütternd“, ${ }^{13}$ und meint damit nicht die engagierte Arbeit des Fähnleins der viereinhalb Aufrechten in der Geschäftsstelle.

Klaus Ceynowa ist zuzustimmen, wenn er in scharfen Worten kritisiert, dass der langfristige Betrieb von Gemeinschaftseinrichtungen regelmäßig an die fachlich ,zuständigen“ Institutionen zurückverwiesen werde. Sie seien aber gar nicht wirklich zuständig, sondern hätten aufgrund ihrer Fachkompetenz nur die Bereitschaft zur projektförmigen Erprobung übergreifender Modelle übernommen:

Diese geraten damit in einen kräftezehrenden Kreislauf von internen und externen Evaluierungen, Begutachtungen und Prüfungen, in denen sie finanzielle „Mehrbedarfe“ zu „rechtfertigen“ haben, obwohl klar ersichtlich ist, dass dieses Mehr nicht den einzelnen

$12 \mathrm{http} / / /$ www.bibliotheksportal.de.

13 Frank Simon-Ritz auf dem Deutschen Bibliothekartag in Bremen 2014. In: Schleh, Bernd: Ohne elektronische Medien geht (fast) nichts. Politische Appelle und fundierte Fachdiskussionen: Mehr als 4100 Teilnehmer beim Bremer Bibliothekartag. In: BuB - Forum Bibliothek und Information 66 (2014) H. 7-8. S. 548-553, hier S. 550. 
Einrichtungen zugutekommt, sondern der anspruchsvollen Aufgabe der nationalen Koordinierung ihrer Aufgaben. ${ }^{14}$

Die Gefahr besteht, dass die kooperationswilligen und -fähigen Bibliotheken irgendwann nur noch ihr eigenes kleines Vorgärtchen bestellen.

Es ist ein Kreuz mit den Gemeinschaftsaufgaben der Bibliotheken! Der Bund darf nicht ohne Weiteres in Vorleistung treten, weil Kultur und Hochschulen Ländersache sind. Aber seit der Grundgesetzänderung vom 1. Januar 2015 hat er in Fällen überregionaler Bedeutung bei der Förderung von Wissenschaft, Forschung und Lehre wieder eine Mitwirkungsmöglichkeit. Vielleicht könnte er mit dieser Kompetenz offensiver umgehen? In erster Linie ist es aber die Gemeinschaft der Länder, die es versäumt, ihre Hausaufgaben zu machen. Das Resultat ist Teilnahmslosigkeit der Politik auf einem zukunftsrelevanten Feld staatlichen Handelns. Damit fehlt nicht nur die Gestaltung eines Politikfelds, sondern auch Streitkultur und Öffentlichkeit, die für eine Demokratie essenziell sind.

Es ist nicht so, dass die aktive Gestaltung der offenen Aufgaben nicht als Problem erkannt worden wäre. Verschiedene Institutionen wie Wissenschaftsrat, Gemeinsame Wissenschaftskonferenz oder Deutsche Forschungsgemeinschaft haben in den letzten Jahren Handlungsfelder zu identifizieren und ein Gesamtkonzept zu entwickeln versucht. Vom Rat für Informationsinfrastrukturen war schon die Rede.

Generell ist jedoch in den Planungspapieren, die von den verschiedenen Gremien in diesem Zusammenhang herausgegeben werden, die Tendenz erkennbar, die digitale Agenda, wie sie im Bereich Naturwissenschaften, Technik und Medizin ansteht, vorschnell auf alle Bereiche zu übertragen. Es besteht die Neigung, die Digitalität auf Kosten der Buchkultur starkzumachen. Die Bedeutung des klassischen Aufgabenspektrums der Bibliotheken für die Kultur- und Geisteswissenschaften sowie für Lehre, Studium und Öffentlichkeit wird gerne unterschätzt - was Wunder, denn es sind Gremien der Forschung, in denen die Papiere entstehen.

Die großen unerledigten Aufgaben auf gesamtstaatlicher Ebene in Deutschland sind:

1 Abgestimmte Sammlungspolitik der wissenschaftlichen Bibliotheken

2 Koordinierte Archivierung der gedruckten Überlieferung und Pflege der Originale

3 Langzeitarchivierung der digitalen Medien

14 Ceynowa, Klaus: „Bibliothekspolitik“- Prätention, Praxis und Perspektiven. In: Bibliothek Forschung und Praxis 40 (2016) H. 3. S. 411-423, hier S. 422. 
4 Aufgabenteilung bei den Forschungsdaten

5 Strategie zur Retrodigitalisierung

6 Schaffung eines starken überregionalen Kompetenzzentrums der Bibliotheken

Die Aufgaben anzupacken, würde, grob gerechnet, gut 100 Millionen Euro pro Jahr kosten. Alle wissenschaftlichen Bibliotheken in Deutschland zusammen verfügen pro Jahr etwas über eine Milliarde Euro an Betriebskosten. Tatsächlich würde der Betrieb von Gemeinschaftseinrichtungen den Steuerzahler mehr Geld kosten, aber nur anfangs. Nach einer Phase der Umstrukturierung müssten nicht mehr finanzielle Mittel ins Spiel kommen als bisher. Die Gemeinschaftsprojekte und -einrichtungen würden es möglich machen, dass die Erwerbungsetats lokaler Bibliotheken entlastet, die Erhaltung der schriftlichen Überlieferung koordiniert angegangen, die Aufgaben der Bibliotheksverbünde besser verteilt sowie vielfache Effizienzgewinne realisiert werden könnten.

In dieser Situation muss die Politik eingreifen und den Bibliotheken bessere Rahmenbedingungen für ihre Arbeit verschaffen. Den Gemeinschaftsleistungen der Bibliotheken muss im Interesse von Wirtschaftlichkeit und Allgemeinwohl der Weg gebahnt werden. Die Umverteilung würde sich wissenschaftspolitisch und volkswirtschaftlich auszahlen - in der Redeweise eines klugen Weimarer Staatsministers gesagt: Sie würde „geräuschlos unberechenbare Zinsen“ spenden. ${ }^{15}$

Zusammengefasst bedeutet dies: Bibliotheken sind ihrem Wesen nach Agenturen der Vernetzung. Aber es fehlen die Akteure für eine übergreifende Bibliothekspolitik. Die Gemeinschaft der Länder hat andere Sorgen oder ist sich uneins. Kein Bundesministerium fühlt sich zuständig. Es gibt auch keine starke eigene Koordinationsinstanz mehr wie früher das Deutsche Bibliotheksinstitut, die an allen Ecken und Enden fehlt. So sitzen die Bibliotheken mit ihren unerledigten Gemeinschaftsaufgaben in der Föderalismusfalle. Als Hochschulbibliotheken sitzen sie auch noch in der Wettbewerbsfalle ihrer eigenen Dachorganisation. Aus den vielen guten Bibliotheken ist noch kein gutes System der Bibliotheken geworden.

Welcher Akteur befreit die Bibliotheken aus ihrer misslichen Situation? Eine nationale Bibliothekspolitik müsste die Informationsinfrastruktur der Zukunft so anzulegen versuchen, dass die überregionalen Aufgaben der Bibliotheken arbeitsteilig wahrgenommen werden können, ohne das Potenzial der regionalen

15 Goethe, Johann Wolfgang: Tag- und Jahres-Hefte [1801]. In: Johann Wolfgang Goethe. Sämtliche Werke. Briefe, Tagebücher und Gespräche. Abt. I, Bd. 17. Hrsg. von Irmtraut Schmid. Frankfurt am Main: Deutscher Klassiker Verlag 1994, S. 77. 
und lokalen Strukturen zu schwächen. ${ }^{16}$ Bei der einzelnen Institution würden Spielräume eröffnet und Kräfte freigesetzt. Ein aufgeklärter Föderalismus wäre die denkbar beste Verfassung der Republik.

Wenn niemand bereit ist, als Akteur für eine nationale Bibliothekspolitik aufzutreten und das Zusammenwirken zu forcieren, bliebe nur wieder der Weg der Selbststeuerung. Damit das halbwegs funktioniert, müssten die Unterhaltsträger den Bibliotheken, aber bitte schön, deutlich mehr Autonomie zubilligen. Dann müssten die Bibliotheken rechtliche Spielräume und finanzielle Mittel bekommen, um Gemeinschaftsprojekte zu finanzieren und Verträge abzuschließen. Dann könnten sie sich ein Stück weit selber helfen. In der Schweiz können Bibliotheken Aktiengesellschaften gründen. ${ }^{17}$ Auf jeden Fall gibt es eine Fülle von Aufgaben für eine neue Generation von ganz vielen kooperationsfreudigen Thomas Bürgers.

16 Vgl. Drees, Bastian: Zukunft der Informationsinfrastrukturen: Das deutsche Bibliothekswesen im digitalen Zeitalter. In: Perspektive Bibliothek 5 (2016) H. 1. S. 25-48. http://dx.doi. org/10.11588/pb.2016.1.31385.

17 Vgl. Niederer, Ulrich u. Dani Tschirren: Die Kooperative Speicherbibliothek Schweiz - Erfahrungen nach einem Jahr Betrieb. In: ABI Technik 37 (2017) H. 2. S. 103-110. 